\title{
Strictly Level-Decreasing Logic Programs
}

\author{
Anthony Karel Seda \\ Department of Mathematics, National University of Ireland \\ Cork, Ireland \\ E-mail: aks@bureau.ucc.ie \\ Pascal Hitzler \\ Mathematische Fakultät, Universität Tübingen \\ Tübingen, Germany \\ E-mail: pascal.hitzler@student.uni-tuebingen.de
}

\begin{abstract}
We study strictly level-decreasing logic programs (sld-programs) as defined earlier by the present authors. It will be seen that sld-programs, unlike most other classes of logic programs, have both a highly intuitive declarative semantics, given as a unique supported model, and are computationally adequate in the sense that every partial recursive function can be represented by some sld-program $P$. Allowing for a safe use of cuts, an interpreter based on SLDNF-resolution, as implemented for example in standard Prolog systems, is shown to be sound and complete with respect to this class of programs. Furthermore, we study connections between topological dynamics and logic programming which are suggested by our approach to the declarative semantics of sld-programs.
\end{abstract}

\section{Introduction}

A programming paradigm consists of a syntax or formal language and an interpreter which assigns a procedural semantics to any program satisfying the given syntactical conditions. The procedural semantics of a given program therefore assigns to any allowed input value one or more output values.

Logic programming is distinguished from other programming paradigms by describing the syntax of a logic program $P$ as a set of clauses from first (or higher) order logic, satisfying some additional properties. Viewing $P$ as a set of axioms, a declarative semantics for $P$ is given by a distinguished model $M$ for $P$. Ideally, $M$ represents the meaning intended by the programmer implementing $P$.

The classical example of this is provided by definite or positive logic programs. In this case, the syntax is simply the Horn-clause subset of first order predicate logic together with SLD-resolution as interpreter, and the declarative semantics is taken to be the least Herbrand model for the given program.

Horn-clauses, however, do not allow negation symbols in their bodies and therefore lack expressiveness. To overcome this, one usually considers normal logic programs, that is to say, programs which consist of a (possibly infinite) set of clauses of the form $A \leftarrow A_{1}, \ldots, A_{k_{1}}, \neg B_{1}, \ldots, \neg B_{l_{1}}$ with $k_{1}, l_{1} \geq 0$, where $A_{i}$ and $B_{j}$ are atoms. For this class of programs the usual interpreter is SLDNF-resolution, see [5].

It turns out to be difficult to assign a declarative semantics to normal logic programs in full generality. Therefore, various subclasses of logic programs have been proposed in attempts to resolve this issue. These subclasses are often defined by conditions on their syntax, and they vary in relation to the difficulty of assigning them a satisfactory declarative semantics. Indeed, it will be convenient next to briefly review certain of these conditions and relate them to the main topic of this paper. In order to do this, we recall the 
standard notation $B_{P}$ for the Herbrand base of a logic program $P$ and the notation $\operatorname{ground}(P)$ for the set of all ground instances of clauses in $P$, see [5, 11].

1.1 Definition A normal logic program $P$ is called (1) acyclic, (2) strictly level-decreasing (sld), (3) locally stratified, respectively, if there exists some countable ordinal $\gamma$ and a mapping $l: B_{P} \rightarrow \gamma$, called a level mapping, such that for every clause $A \leftarrow A_{1}, \ldots, A_{k_{1}}, \neg B_{1}, \ldots, \neg B_{l_{1}}$ in $\operatorname{ground}(P)$ and for all $i, j$ we have (1) $\gamma=\omega$ and $l\left(A_{i}\right), l\left(B_{j}\right)<l(A)$, (2) $l\left(A_{i}\right), l\left(B_{j}\right)<l(A),(3) l\left(A_{i}\right) \leq l(A)$ and $l\left(B_{j}\right)<l(A)$, respectively.

It is clear that every acyclic program is strictly level-decreasing and that every strictly level-decreasing program is locally stratified. In fact, the terminology "semi-strictly level-decreasing" was used in place of "locally stratified" in [11], but will not be used here.

Every acyclic program $P$ has a unique supported model $M$ (see [3]). Moreover, it was shown in [1] that SLDNF-resolution as interpreter terminates on all input values. So for acyclic programs, declarative and procedural semantics agree. But, since SLDNF-resolution always terminates, it is not possible to represent every partial recursive function as an acyclic program. The class of all acyclic programs is therefore not computationally adequate.

On the other hand, locally stratified programs do not have unique supported models, but each such program does have a unique perfect model as defined and constructed in [8]. Furthermore, the class of all locally stratified programs contains the class of all definite programs and is therefore computationally adequate since the latter class has this property, see [13]. To elaborate a little further on the issues involved, let $P$ be the following (locally stratified) program

$$
\begin{aligned}
& p(0) \leftarrow \neg q(0) \\
& q(0) \leftarrow q(0)
\end{aligned}
$$

Here, we have two minimal and supported models $\{p(0)\}$ and $\{q(0)\}$ for $P$, and it is natural to try to find a way of showing that one is preferable to the other in some sense. In [8] it is argued that the perfect model provides such a way, and in fact for the program in question the perfect model semantics coincides with the first model, $\{p(0)\}$, which may therefore be viewed as preferable to the second model, $\{q(0)\}$. On the other hand, both are models of the Clark-completion $\operatorname{comp}(P)$ of $P$, due to the second clause in the progam $P$. It could in fact be argued that if a programmer explicitly implements this second clause, he intends to allow $\{q(0)\}$ as a model for the program, which is consistent with the view of $\operatorname{comp}(P)$ as a set of first-order clauses. The perfect model semantics ignores this second clause and assumes that the programmer did not intend the logical meaning underlying it. These points hint at some of the problems involved in finding a satisfactory semantics for locally stratified programs. From the procedural point of view, a practically implementable interpreter which can deal with the power of expressiveness of locally stratified programs remains to be found. Nevertheless, it is safe to say that locally stratified programs form one of the most important classes of programs in AI and in the study of disjunctive databases.

The class of all strictly level-decreasing programs, however, does not have the undesirable properties of the other two classes of programs just discussed. Indeed, it was shown in [11] that every sld-program has a unique supported model, i.e. the completion $\operatorname{comp}(P)$ of $P$ has a unique model. Moreover, it was shown how this model can be constructed by a transfinite sequence. It will be shown here that the class of sld-programs is computationally adequate, and indeed the structure of the paper is as follows.

Section 2 of the present paper is devoted to the declarative and procedural semantics of sld-programs. We briefly review the results already obtained in [11] and investigate in Section 2.1 simple methods to construct 
the unique supported model. In Section 2.2, we will allow the use of safe cuts in the syntax of sld-programs and show that these together with an interpreter based on SLDNF-resolution, as implemented in standard Prolog systems, can represent every partial recursive function.

Our approach to the declarative semantics of sld-programs employs methods which suggest connections between logic programming and topological dynamics. In Section 3, we therefore investigate these and connect them up with our previous observations on this topic presented in [11]. In particular, in Sections 3.1 and 3.2 we study the Vietoris space of $B_{P}$ and iterated function systems in the context of arbitrary normal logic programs. These topics, i.e. Vietoris space and iterated function systems, have both been brought into prominence by the work of Abbas Edalat, see [2]. Finally, we conclude with some further observations on sld-programs which relate them yet more closely to topological dynamics. Our overall claim is that the class of strictly level-decreasing programs is particularly interesting, both computationally and mathematically, and the objective of this paper is to substantiate this claim.

\section{Semantics of Strictly Level-Decreasing Logic Programs}

It will be convenient to recall first some basic notions used in the sequel. Details and further background can be found in [5].

Let $P$ denote a normal logic program with underlying first order language $\mathcal{L}$. As usual, $U_{P}$ and $B_{P}$ will denote respectively the Herbrand universe and Herbrand base of $P$, and $I_{P}$ or $\mathcal{P}\left(B_{P}\right)$ will denote the set of all Herbrand interpretations for $\mathcal{L}$ (or for $P$ ). We let $T_{P}: I_{P} \rightarrow I_{P}$ denote the usual immediate consequence operator associated with $P$. Throughout, $l$ will denote a level mapping; thus $l$ is simply a mapping $l: B \rightarrow \gamma$, where $B$ is a set and $\gamma$ is an arbitrary countable ordinal (usually, $B$ will be the Herbrand base $B_{P}$ ). For any such mapping, we set $\mathcal{L}_{\alpha}=\{a \in B ; l(a)<\alpha\}$ and let $\Gamma_{\gamma}$ be the set of symbols $\left\{2^{-\alpha} ; \alpha<\gamma\right\}$ ordered by $2^{-\alpha}<2^{-\beta}$ if and only if $\beta<\alpha$. (In the case $B=B_{P}$, we will denote a typical element of $B_{P}$, i.e. a ground atom, by $A$ rather than by the lower case $a$ ).

Given the set $B$, the Cantor topology $Q$ on $D=2^{B}$ is characterized via convergence as follows: a net $I_{\lambda}$ converges in $Q$ if and only if every $a \in B$ is either eventually in $I_{\lambda}$ or eventually not in $I_{\lambda}$. In particular, this is so when $B$ is countable (for example when $B=B_{P}$ ), in which case sequences suffice to describe topological concepts and the stated convergence criterion still applies. This fact, which we will refer to as the convergence criterion, will be used in several places and is recorded as Proposition 4 in [9]. Finally, for a net $I_{\lambda}$, let $\operatorname{gl}\left(I_{\lambda}\right)$ denote the greatest limit of $I_{\lambda}$ in the Scott topology on $D$, see [9]. It was shown in [10] that if $I_{\lambda} \rightarrow I$ in $Q$, then $I=\operatorname{gl}\left(I_{\lambda}\right)$ and that $\operatorname{gl}\left(I_{\lambda}\right)=\left\{a \in B ; a \in I_{\lambda}\right.$ eventually $\}$. The Cantor topology and its rôle in logic programming was studied in $[9,10]$ and in $[11,12]$.

In [11], it was shown that every sld-program $P$ has a unique supported model $M_{P}$ which can therefore be understood to be the standard semantics for $P$. In order to make this paper relatively self-contained, we briefly review the main points of our construction of $M_{P}$ in [11], and refer the reader to that paper for details.

Let $P$ denote a normal logic program which is locally stratified with respect to a level mapping $l: B_{P} \rightarrow$ $\gamma$, where $\gamma \geq 1$. For each $n$ satisfying $0<n \leq \gamma$, let $P_{[n]}$ denote the set of all clauses in ground $(P)$ in which only atoms $A$ with $l(A)<n$ occur. We define $T_{[n]}: \mathcal{P}\left(\mathcal{L}_{n}\right) \rightarrow \mathcal{P}\left(\mathcal{L}_{n}\right)$ by $T_{[n]}(I)=T_{P_{[n]}}(I)$. Next, we construct the transfinite sequence $\left(I_{n}\right)_{n \in \gamma}$ inductively as follows. For each $m \in N$, we put $I_{[1, m]}=T_{[1]}^{m}(\emptyset)$ and set $I_{1}=\bigcup_{m=0}^{\infty} I_{[1, m]}$. If $n \in \gamma$, where $n>1$ is a successor ordinal, then for each $m \in N$ we put $I_{[n, m]}=T_{[n]}^{m}\left(I_{n-1}\right)$ and set $I_{n}=\bigcup_{m=0}^{\infty} I_{[n, m]}$. If $n \in \gamma$ is a limit ordinal, we put $I_{n}=\bigcup_{m<n} I_{m}$. Finally, we put $I_{[P]}=M_{P}=\bigcup_{n<\gamma} I_{n}$.

Next, we recall a construction related to domain theory which we made in [11]. Let $B$ be a countable 
set and let $l: B \rightarrow \gamma$ be a level mapping. It is well-known that $D=2^{B}$ is a domain with respect to set inclusion. The level mapping $l$ induces a distance function $d_{l}=d: D \times D \rightarrow \Gamma_{\gamma+1}$ via $d(I, J)=$ $\min \left\{2^{-\alpha} ; a \in I\right.$ if and only if $a \in J$ for all $a \in B$ with $\left.l(a)<\alpha\right\}$. This distance function is in fact a generalized ultrametric as defined in [6], i.e. it satisfies the following conditions, where we denote $2^{-\gamma}$ by 0 :

(1) $d(I, I)=0$ for all $I \in D$.

(2) $d(I, J)=d(J, I)$ for all $I, J \in D$.

(3) If $d(I, J) \leq \alpha$ and $d(J, K) \leq \alpha$, then $d(I, K) \leq \alpha$.

Now let $P$ be a program which is strictly level-decreasing with respect to some level mapping $l$ and take $B=B_{P}$. Then $l$ induces a generalized ultrametric on $I_{P}$, and $T_{P}$ is strictly contracting, i.e. it satisfies $d\left(T_{P}(I), T_{P}(J)\right)<d(I, J)$ for all $I, J \in I_{P}$. Moreover, $I_{P}$ and $T_{P}$ satisfy the hypotheses of the theorem of Priess-Crampe and Ribenboim (see $[6,7]$ ) which therefore yields the existence of a unique fixed point of $T_{P}$ and therefore of a unique supported model for $P$.

\subsection{Declarative Semantics}

We begin our study of sld-programs by showing how such a program $P$ can be endowed with a canonical level mapping $l_{P}$ which is smallest in a certain obvious sense.

2.1 Definition Let $P$ be a program which is strictly level-decreasing with respect to a level mapping $l$. We define a level mapping $l_{P}$ on $B_{P}$ as follows. For every $A \in B_{P}$ which does not occur as a head in ground $(P)$, let $l_{P}(A)=0$. For every $A \in B_{P}$ which occurs as the head of a unit clause but not as the head of any non-unit clause, let $l_{P}(A)=0$. Now let $A \in B_{P}$ be such that $A$ is the head of some clause(s) in ground $(P)$. Let $\mathcal{B}_{A}$ be the collection of body-literals occurring in these clauses. Note that $\mathcal{B}_{A}$ is finite for every $A$ if $P$ has no local variables. Now suppose that for every $B \in \mathcal{B}_{A}, l_{P}(B)$ is already defined. Let $M_{A}=\sup _{B \in \mathcal{B}_{A}} l_{P}(B)$ and set $l_{P}(A)=M_{A}+1$, if $M_{A}$ is a successor ordinal, and set $l_{P}(A)=M_{A}$, if $M_{A}$ is a limit ordinal. Then $l_{P}$ is obtained by transfinitely iterating this procedure. We will refer to $l_{P}$, as defined above, as the (canonical) level mapping of $P$ and, further, $\gamma_{P}$ will denote the smallest ordinal $\alpha$ such that $l_{P}(A) \in \alpha$ for all $A \in B_{P}$.

2.2 Proposition Let $P$ be a program which is strictly level-decreasing with respect to some level mapping $l$. Then $l_{P}$, as defined above, is a function on $B_{P}$ and $P$ is strictly level-decreasing with respect to $l_{P}$. Moreover, if $P$ has no local variables, then $\gamma_{P} \leq \omega$ and hence $P$ is acyclic ${ }^{1}$ and acceptable ${ }^{1}$.

Proof: First we show that $\operatorname{dom}\left(l_{P}\right)=B_{P}$. Suppose there is $A \in B_{P} \backslash \operatorname{dom}\left(l_{P}\right)$; we can further suppose that $l(A)$ is minimal for $A$ with this property. Then there must be some $B \in \mathcal{B}_{A}$ with $B \notin \operatorname{dom}\left(l_{P}\right)$, otherwise $l_{P}(A)$ is defined in the process given in Definition 2.1. Since $P$ is an sld-program, we have $l(B)<l(A)$ which contradicts the choice of $A$ with $l(A)$ minimal. Therefore, $l_{P}$ is a level mapping, and obviously $P$ is strictly level-decreasing with respect to it. Finally, if $P$ has no local variables, then the set $\mathcal{B}_{A}$ is finite for every $A \in B_{P}$, and so $l_{P}$ maps into $\omega$. Hence, $\gamma_{P} \leq \omega$.

The construction above of the level mapping $l_{P}$ can be used to determine whether or not a given program $P$ is strictly level-decreasing, and the following corollary is immediate.

\footnotetext{
${ }^{1}$ See [1].
} 
2.3 Corollary Let $P$ be an arbitrary normal logic program. Then $P$ is strictly level-decreasing if and only if $\operatorname{dom}\left(l_{P}\right)=B_{P}$.

2.4 Proposition Let $P$ be a program which is strictly level-decreasing with respect to a level mapping $l$. Then for every $A \in B_{P}$, we have $l_{P}(A) \leq l(A)$.

Proof: Suppose the conclusion is false. Thus, there is $A \in B_{P}$ with $l(A)<l_{P}(A)$, and such that $l(A)$ is minimal. Then, for all $B \in \mathcal{B}_{A}$, we have $l(B)<l(A)$ because $P$ is strictly level-decreasing. Therefore, by minimality of $l(A)$, we have $l(B) \geq l_{P}(B)$ for all $B \in \mathcal{B}_{A}$. By definition of $l_{P}$, we see that $l_{P}(A)=$ $\min \left\{\alpha ; \alpha>l_{P}(B), B \in \mathcal{B}_{A}\right\} \leq \min \left\{\alpha ; \alpha>l(B), B \in \mathcal{B}_{A}\right\} \leq l(A)$. From this we obtain $l_{P}(A) \leq l(A)$, giving the required contradiction.

As stated in the introduction to this section, a procedure for finding the perfect model $M_{P}$ for locally stratified programs $P$ was given by us in [11]. Our approach shed light on the original construction made by Przymusinski in [8], and involved studying convergence in $Q$ of certain transfinite sequences of iterates which were carefully controlled in relation to the level mapping associated with the local stratification. One particular fact which emerged from that study, see [11, Theorem 5], was that for acyclic programs $P, M_{P}$ can be realised as the limit in $Q$ of the sequence $T_{P}^{n}(I)$ for any choice of $I \in I_{P}$ (in particular, the choice of $I=\emptyset$ is especially natural). The question arises as to whether or not this result can be generalized to programs which are strictly level-decreasing relative to an arbitrary level mapping. The following example shows that the answer to this question is negative.

2.5 Example Consider the following program $P$ :

$$
\begin{aligned}
t(0) & \leftarrow p(X) \\
p(s(X)) & \leftarrow r(X) \\
r(s(X)) & \leftarrow p(X) \\
p(0) & \leftarrow \neg q(0) \\
q(0) & \leftarrow
\end{aligned}
$$

and define the level mapping $l$ on $B_{P}$ by: $q\left(s^{n}(0)\right) \mapsto 0, p\left(s^{n}(0)\right) \mapsto n+1, r\left(s^{n}(0)\right) \mapsto n+$ 1 , and $t\left(s^{n}(0)\right) \mapsto \omega$. Then it is clear that $P$ is strictly level-decreasing with respect to $l$. However, on computing the elements of the sequence $T_{P}^{n}(\emptyset)$, we find that $t(0)$ belongs to infinitely many of the $T_{P}^{n}(\emptyset)$ and does not belong to infinitely many others. Therefore, by the convergence criterion, the sequence $T_{P}^{n}(\emptyset)$ does not converge in $Q$ and we see that $M_{P}$ cannot be computed by simply iterating $T_{P}$, by contrast with the case of $\omega$-level mappings.

Nevertheless, considerable simplification does arise in constructing $M_{P}$ for sld-programs as distinct from general locally stratified programs, and the remaining results of this section demonstrate this fact; much of this simplification ultimately rests on the recursion equations in [11, Corollary 2]. For the rest of this section, unless otherwise stated, $P$ will denote an arbitrary sld-program with level mapping $l$, and $T$ will denote $T_{P}$.

2.6 Lemma Suppose that $P$ is a locally stratified normal logic program. Then for any ordinal $n \geq 1$ and subset $J \subseteq B_{P}$, we have:

(1) $T_{[n]}\left(J \cap \mathcal{L}_{n}\right)=T_{[n]}(J)$, and 
(2) $T_{P(n)}\left(J \cap \mathcal{L}_{n+1}\right)=T_{P(n)}(J)$.

If, further, $P$ is strictly level-decreasing, then we also have:

(3) $T_{[n+1]}\left(J \cap \mathcal{L}_{n}\right)=T_{[n+1]}(J)$, and

(4) $T_{P(n)}\left(J \cap \mathcal{L}_{n}\right)=T_{P(n)}(J)$.

Proof: (1) Suppose that $A \in T_{[n]}\left(J \cap \mathcal{L}_{n}\right)$. Then there is a clause in $P_{[n]}$ of the form $A \leftarrow$ $A_{1}, \ldots, A_{k_{1}}, \neg B_{1}, \ldots, \neg B_{l_{1}}$ such that $A_{1}, \ldots, A_{k_{1}} \in J \cap \mathcal{L}_{n}$ and $B_{1}, \ldots, B_{l_{1}} \notin J \cap \mathcal{L}_{n}$. Since $l(A)<n$, we have $l\left(A_{i}\right), l\left(B_{j}\right)<n$ for all $i, j$ and so $A_{1}, \ldots, A_{k_{1}}, B_{1}, \ldots, B_{l_{1}} \in \mathcal{L}_{n}$. Therefore, $A_{1}, \ldots, A_{k_{1}} \in J$ and $B_{1}, \ldots, B_{l_{1}} \notin J$ from which we obtain that $A \in T_{[n]}(J)$.

Conversely, suppose that $A \in T_{[n]}(J)$. Then there is a clause in $P_{[n]}$ of the form $A \leftarrow$ $A_{1}, \ldots, A_{k_{1}}, \neg B_{1}, \ldots, \neg B_{l_{1}}$ such that $A_{1}, \ldots, A_{k_{1}} \in J$ and $B_{1}, \ldots, B_{l_{1}} \notin J$. By level considerations again, we have $A_{1}, \ldots, A_{k_{1}} \in J \cap \mathcal{L}_{n}$ and $B_{1}, \ldots, B_{l_{1}} \notin J \cap \mathcal{L}_{n}$. Therefore, $A \in T_{[n]}\left(J \cap \mathcal{L}_{n}\right)$.

The remaining statements are proved similarly taking note of the appropriate equalities and inequalities between the values $l(A)$ and $l\left(A_{i}\right), l\left(B_{j}\right)$ for the atoms occurring in a typical clause $A \leftarrow$ $A_{1}, \ldots, A_{k_{1}}, \neg B_{1}, \ldots, \neg B_{l_{1}}$ in $P_{[n]}$ and in $P(n)$.

It will be convenient to recall next a definition made in [4, Definition 7.11] for a normal logic program $P$. Again writing $T$ for $T_{P}$, we define $T^{(n)}: \mathcal{P}\left(\mathcal{L}_{n}\right) \rightarrow \mathcal{P}\left(\mathcal{L}_{n}\right)$ by $T^{(n)}(I)=T(I) \cap \mathcal{L}_{n}$, where $n$ is an arbitrary ordinal satisfying $1 \leq n \leq \gamma$. In fact, this statement differs slightly from that given in [4] to the extent that $\mathcal{L}_{n}$ as used here differs slightly from its meaning in [4].

2.7 Lemma Suppose that $P$ is a locally stratified normal logic program. Then the following statements hold.

(1) For any subset $I \subseteq B_{P}$ and any ordinal satisfying $1 \leq n \leq \gamma$, we have $T_{[n]}(I)=T^{(n)}(I)\left(=T(I) \cap \mathcal{L}_{n}\right)$.

(2) If, further, $P$ is strictly level-decreasing, then for any ordinal $n$ satisfying $1 \leq n \leq \gamma$, we have $I_{n+1}=$ $I_{n} \cup T_{P(n)}\left(I_{n}\right)=T_{[n+1]}\left(I_{n}\right)$.

Proof: Statement (1) is simply a reiteration of [4, Proposition 7.17] allowing for the difference, already mentioned, between the meaning of $\mathcal{L}_{n}$ as used here and in [4].

For (2), we have $I_{n+1}=\bigcup_{m=0}^{\infty} I_{[n+1, m]}=\bigcup_{m=0}^{\infty} I_{n} \cup T_{P(n)}\left(I_{n}\right)$ by [11, Corollary 2]. Therefore, $I_{n+1}=I_{n} \cup T_{P(n)}\left(I_{n}\right)=T_{[n]}\left(I_{n}\right) \cup T_{P(n)}\left(I_{n}\right)=T_{[n+1]}\left(I_{n}\right)$, using the fact that $I_{n}$ is a fixed point of $T_{[n]}$.

2.8 Theorem Suppose that $P$ is a strictly level-decreasing normal logic program. Then for every limit ordinal $\alpha$ and $k \in \mathbb{N}$ we have $T^{k}\left(I_{\alpha}\right) \cap \mathcal{L}_{\alpha+k}=I_{\alpha+k}$, and for $\beta=\alpha+\omega$ we have $\lim _{n \in \mathbb{N}}\left(T_{[\beta]}\right)^{n}\left(I_{\alpha}\right)=I_{\beta}$ in $Q$.

Proof: The first statement follows from Lemmas 2.6 and 2.7 by induction. Indeed, for $k=0$, the statement obviously holds on noting that $I_{\alpha} \subseteq \mathcal{L}_{\alpha}$. Now suppose the statement holds for some $k \geq 0$. Then by Lemma 2.7 (2), the induction hypothesis, Lemma 2.6 (1) and (4), and Lemma 2.7 (1) applied in that order we have $I_{\alpha+k+1}=T_{[\alpha+k+1]}\left(I_{\alpha+k}\right)=T_{[\alpha+k+1]}\left(T^{k}\left(I_{\alpha}\right) \cap \mathcal{L}_{\alpha+k}\right)=T_{[\alpha+k]}\left(T^{k}\left(I_{\alpha}\right) \cap\right.$ $\left.\left.\left.\mathcal{L}_{\alpha+k}\right) \cup T_{P(\alpha+k)}\left(T^{k}\left(I_{\alpha}\right) \cap \mathcal{L}_{\alpha+k}\right)\right)=T_{[\alpha+k]}\left(T^{k}\left(I_{\alpha}\right)\right) \cup T_{P(\alpha+k)}\left(T^{k}\left(I_{\alpha}\right)\right)\right)=T_{[\alpha+k+1]}\left(T^{k}\left(I_{\alpha}\right)\right)=$ $T\left(T^{k}\left(I_{\alpha}\right)\right) \cap \mathcal{L}_{\alpha+k+1}=T^{k+1}\left(I_{\alpha}\right) \cap \mathcal{L}_{\alpha+k+1}$.

For the second statement, we have $\left(T_{[\beta]}\right)^{n}\left(I_{\alpha}\right) \cap \mathcal{L}_{\alpha+n}=I_{\alpha+n}$ for all $n \in \mathbb{N}$ by induction on $n$. Indeed, the statement clearly holds for $n=0$. Now suppose it holds for some $n \geq 0$. Then, using Lemma 2.7 (1), Lemma 2.6 (3), the induction hypothesis and Lemma 2.7 (2), we have $\left(T_{[\beta]}\right)^{n+1}\left(I_{\alpha}\right) \cap$ 
$\mathcal{L}_{\alpha+n+1}=T_{[\beta]}\left(\left(T_{[\beta]}\right)^{n}\left(I_{\alpha}\right)\right) \cap \mathcal{L}_{\alpha+n+1}=T\left(\left(T_{[\beta]}\right)^{n}\left(I_{\alpha}\right)\right) \cap \mathcal{L}_{\beta} \cap \mathcal{L}_{\alpha+n+1}=T\left(\left(T_{[\beta]}\right)^{n}\left(I_{\alpha}\right)\right) \cap \mathcal{L}_{\alpha+n+1}=$ $T_{[\alpha+n+1]}\left(\left(T_{[\beta]}\right)^{n}\left(I_{\alpha}\right)\right)=T_{[\alpha+n+1]}\left(\left(T_{[\beta]}\right)^{n}\left(I_{\alpha}\right) \cap \mathcal{L}_{\alpha+n}\right)=T_{[\alpha+n+1]}\left(I_{\alpha+n}\right)=I_{\alpha+n+1}$. Now let $A \in B_{P}$ be arbitrary. Then $l(A)=\alpha+m_{0}$ for some limit ordinal $\alpha \geq 0$ and some $m_{0} \in \mathbb{N}$. Thus, $A \in \mathcal{L}_{\alpha+m_{0}+1}$ for some $\alpha$ and $m_{0}$. Now either $A \in M_{P}$ or $A \notin M_{P}$, where $M_{P}=\bigcup_{n<\gamma} I_{n}$. If $A \in M_{P}$, then we must have $A \in I_{\alpha+m_{0}+1}$ by [11, Lemma 1], otherwise $A \notin M_{P}$. Hence, $A \in\left(T_{[\beta]}\right)^{m_{0}+1}\left(I_{\alpha}\right)$ and therefore $A \in\left(T_{[\beta]}\right)^{m}\left(I_{\alpha}\right)$ eventually. If $A \notin M_{P}$, then $A$ is eventually not in $\left(T_{[\beta]}\right)^{m}\left(I_{\alpha}\right)$. Hence, by the convergence criterion, $\left(T_{[\beta]}\right)^{m}\left(I_{\alpha}\right)$ converges in $Q$ and obviously converges to $I_{\beta}$.

The result just obtained allows us to further simplify the calculation of $M_{P}$, and to do this it will be convenient to establish the following terminology.

2.9 Definition Let $P$ be a strictly level decreasing-program. For every limit ordinal $0 \leq \alpha<\gamma_{P}$ and $\beta=\alpha+\omega$ the next limit ordinal, we define $P_{\beta}$ to be the set of all clauses in ground $(P)$ with head $A$ such that $\alpha \leq l(A)<\beta$. The usual order on the limit ordinals induces an order on the set of all $P_{\beta}$. We call each $P_{\beta}$ an $\omega$-component of $P$.

2.10 Lemma Suppose that $P$ is a strictly level-decreasing program, let $\alpha<\gamma_{P}$ be a limit ordinal and let $\beta=\alpha+\omega$. Then for every $n \in \mathbb{N}$, we have $I_{\alpha}=T_{[\alpha]}\left(\left(T_{[\beta]}\right)^{n}\left(I_{\alpha}\right)\right)$.

Proof: We have to show that $d_{l}\left(I_{\alpha},\left(T_{[\beta]}\right)^{n}\left(I_{\alpha}\right)\right) \leq 2^{-\alpha}$ which is obviously true for $n=0$. Suppose it holds for some $n \geq 0$. Since $T_{[\beta]}=T_{P_{\beta}} \cup T_{[\alpha]}$ and since $I_{\alpha}$ is a fixed point of $T_{[\alpha]}$, we have $d_{l}\left(I_{\alpha}, T_{[\beta]}\left(I_{\alpha}\right)\right) \leq 2^{-\alpha}$. Since $T_{[\beta]}$ is strictly contracting with respect to $d_{l}$, we have $d_{l}\left(T_{[\beta]}\left(I_{\alpha}\right),\left(T_{[\beta]}\right)^{n+1}\left(I_{\alpha}\right)\right)<d_{l}\left(I_{\alpha},\left(T_{[\beta]}\right)^{n}\left(I_{\alpha}\right)\right)$. By the strong triangle inequality we get $d_{l}\left(I_{\alpha},\left(T_{[\beta]}\right)^{(n+1)}\left(I_{\alpha}\right)\right) \leq \max \left\{2^{-\alpha}, d_{l}\left(I_{\alpha},\left(T_{[\beta]}\right)^{n}\left(I_{\alpha}\right)\right)\right\}$ and inductively $d_{l}\left(I_{\alpha},\left(T_{[\beta]}\right)^{n+1}\left(I_{\alpha}\right)\right) \leq$ $d_{l}\left(I_{\alpha}, I_{\alpha}\right) \leq 2^{-\alpha}$.

We have the following theorem, where we recall from [4, Definition 7.11] that $T \Uparrow n(I)$ is defined inductively for any $I \in I_{P}$ by setting $T \Uparrow 0(I)=I$ and $T \Uparrow(n+1)(I)=T(T \Uparrow n(I)) \cup I$ for $n \geq 0$.

2.11 Theorem Let $P$ be a strictly level-decreasing program. Then for every limit ordinal $\alpha<\gamma_{P}$ and $\beta=\alpha+\omega$, we have $I_{\beta}=\lim _{n} T_{P_{\beta}} \Uparrow n\left(I_{\alpha}\right)$ in $Q$.

Proof: The statement follows from Theorem 2.8 by the observation that

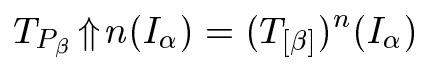

for every $n \in \mathbb{N}$, which we will prove by induction on $n$. Indeed, Equation (1) holds for $n=0$. So suppose it holds for some $n \in \mathbb{N}$. Then, by the induction hypothesis and the previous lemma, we have $T_{P_{\beta}} \Uparrow$ $(n+1)\left(I_{\alpha}\right)=T_{P_{\beta}}\left(T_{P_{\beta}} \Uparrow n\left(I_{\alpha}\right)\right) \cup I_{\alpha}=T_{P_{\beta}}\left(\left(T_{[\beta]}\right)^{n}\left(I_{\alpha}\right)\right) \cup I_{\alpha}=T_{P_{\beta}}\left(\left(T_{[\beta]}\right)^{n}\left(I_{\alpha}\right)\right) \cup T_{[\alpha]}\left(\left(T_{[\beta]}\right)^{n}\left(I_{\alpha}\right)\right)=$ $T_{[\beta]}\left(\left(T_{[\beta]}\right)^{n}\left(I_{\alpha}\right)\right)=\left(T_{[\beta]}\right)^{n+1}\left(I_{\alpha}\right)$ which establishes Equation (1) for every $n \in \mathbb{N}$.

If we put $I_{\beta}=\bigcup_{\alpha<\beta} I_{\alpha}$ for every limit ordinal $\beta$ which is not of the form used in the theorem, then we obtain a method for calculating $M_{P}$. Indeed, we will next give a proof of the fact that every sld-program has a unique supported model. The proof is independent of the results in [11].

Let $P$ be an sld-program. Let $T_{\omega}: \mathcal{P}\left(\mathcal{L}_{\omega}\right) \rightarrow \mathcal{P}\left(\mathcal{L}_{\omega}\right)$ be defined by $T_{\omega}=T_{P_{\omega}}\left(=T_{[\omega]}\right)$. Then, by [11, Theorem 5], $T_{\omega}$ is a contraction with contractivity factor at most $\frac{1}{2}$ with respect to Fitting's complete ultrametric, and therefore has a unique fixed point $J_{\omega}$. (Fitting in [3] defined a complete ultrametric $d$ 
determined by a level mapping $l: B_{P} \rightarrow \omega$ as follows: if $I_{1}=I_{2}$, we set $d\left(I_{1}, I_{2}\right)=0$, otherwise we set $d\left(I_{1}, I_{2}\right)=2^{-n}$, where $I_{1}$ and $I_{2}$ differ on some ground atom of level $n$ but agree on all atoms of lower level).

For every limit ordinal $\alpha>0$ and $\beta=\alpha+\omega$, define inductively $T_{\beta}: \mathcal{P}\left(\mathcal{L}_{\beta}\right) \rightarrow \mathcal{P}\left(\mathcal{L}_{\beta}\right)$ by $T_{\beta}(I)=$ $T_{P_{\beta}}(I) \cup J_{\alpha}$. We show next that $T_{\beta}$ is a contraction with contractivity factor at most $\frac{1}{2}$. To do this we define the level mapping $l_{\beta}: \mathcal{L}_{\beta} \rightarrow \omega$ by $l_{\beta}(A)=0$ if $l(A)<\alpha$, and $l_{\beta}(A)=1+(l(A)-\alpha)$ if $\alpha \leq l(A)<\beta$. Now let $d_{\beta}$ denote Fitting's ultrametric determined by $l_{\beta}$. Notice that $P_{\beta}$ is strictly level-decreasing with respect to $l_{\beta}$ but that $P_{[\alpha]}$ does not have this property.

2.12 Lemma With the notation just established, the mapping $T_{\beta}$ is a contraction with respect to $d_{\beta}$.

Proof: Suppose that $I_{1}$ and $I_{2}$ are arbitrary elements of $\mathcal{P}\left(\mathcal{L}_{\beta}\right)$ and that $d_{\beta}\left(I_{1}, I_{2}\right)=2^{-n}$, where $n \geq 0$. Case $1 . n=0$.

In this case, $I_{1}, I_{2}$ differ on some element of $\mathcal{L}_{\beta}$ of $l_{\beta}$-level 0 . Let $A \in \mathcal{L}_{\beta}$ satisfy $l_{\beta}(A)=0$, but otherwise be arbitrary; then $A \in \mathcal{L}_{\alpha}$. If $A \in J_{\alpha}$, then $A \in T_{\beta}\left(I_{1}\right)$ and $A \in T_{\beta}\left(I_{2}\right)$; if $A \notin J_{\alpha}$, then $A \notin T_{\beta}\left(I_{1}\right)$ and $A \notin T_{\beta}\left(I_{2}\right)$. Thus, $T_{\beta}\left(I_{1}\right)$ and $T_{\beta}\left(I_{2}\right)$ agree on all atoms of $l_{\beta}$-level 0 . Therefore,

$$
d_{\beta}\left(T_{\beta}\left(I_{1}\right), T_{\beta}\left(I_{2}\right)\right) \leq \frac{1}{2}=\frac{1}{2} d_{\beta}\left(I_{1}, I_{2}\right) .
$$

Case 2. $n>0$.

In this case, $I_{1}$ and $I_{2}$ differ on some ground atom of $l_{\beta}$-level $n$ but agree on all ground atoms of lower $l_{\beta}$-level. Let $A \in \mathcal{L}_{\beta}$ be arbitrary with $l_{\beta}(A) \leq n$. Suppose further that $A \in T_{\beta}\left(I_{1}\right)$. If $l_{\beta}(A)=0$, then $A \in \mathcal{L}_{\alpha}$ and hence $A \in J_{\alpha}$. Therefore, $A \in T_{\beta}\left(I_{2}\right)$ also. On the other hand, if $l_{\beta}(A)>0$, then there is a clause $A \leftarrow A_{1}, \ldots, A_{k_{1}}, \neg B_{1}, \ldots, \neg B_{l_{1}}$ in $P_{\beta}$, with $k_{1} \geq 0$ and $l_{1} \geq 0$, which satisfies $I_{1} \models A_{1} \wedge \cdots \wedge$ $A_{k_{1}} \wedge \neg B_{1} \wedge \cdots \wedge \neg B_{l_{1}}$. If $k_{1}=0=l_{1}$, then the clause in question is a unit clause and immediately we have $A \in T_{\beta}\left(I_{2}\right)$. If $k_{1} \neq 0$ or $l_{1} \neq 0$ or both, then $I_{1}$ and $I_{2}$ agree on the atoms $A_{1}, \ldots, A_{k_{1}}, B_{1}, \ldots, B_{l_{1}}$ since their $l_{\beta}$-levels are lower than $n$. Therefore, $I_{2}=A_{1} \wedge \cdots \wedge A_{k_{1}} \wedge \neg B_{1} \wedge \cdots \wedge \neg B_{l_{1}}$ and we have $A \in T_{\beta}\left(I_{2}\right)$. The converse argument holds similarly and so we see that $T_{\beta}\left(I_{1}\right)$ and $T_{\beta}\left(I_{2}\right)$ agree on all ground atoms of $l_{\beta}$-level $\leq n$. Therefore,

$$
d_{\beta}\left(T_{\beta}\left(I_{1}\right), T_{\beta}\left(I_{2}\right)\right) \leq 2^{-(n+1)}=\frac{1}{2} d_{\beta}\left(I_{1}, I_{2}\right)
$$

as required.

It follows from the previous lemma that $T_{\beta}$ has a unique fixed point $J_{\beta}$. For every limit ordinal $\beta$ which is not of the form $\alpha+\omega$ for any limit ordinal $\alpha$, we set $J_{\beta}=\bigcup_{\alpha<\beta} J_{\alpha}$, where the $\alpha$ are limit ordinals. The resulting transfinite sequence $\left(J_{\alpha}\right)$ is increasing since for $\beta=\alpha+\omega$, where $\alpha$ is some limit ordinal, $J_{\beta}$ satisfies $J_{\beta}=T_{\beta}\left(J_{\beta}\right)=T_{P_{\beta}}\left(J_{\beta}\right) \cup J_{\alpha}$, so that $J_{\beta} \supseteq J_{\alpha}$. Finally, on taking $\beta$ to be a limit ordinal such that $\gamma_{P} \leq \beta$, we obtain $J_{\beta}=M_{P}$ i.e. the unique fixed point of $T_{P}$.

\subsection{Procedural Semantics}

For convenience, we establish the following notation for every sld-program $P$. For $A \in B_{P}$, we say that $P \mid=A$ if and only if $A \in M_{P}$. We say that $P \vdash_{\text {SLDNF }} A$ if and only if there is an SLDNF-derivation for $P \cup\{\leftarrow A\}$. 
2.13 Theorem Let $P$ be a strictly level-decreasing program and $A \in B_{P}$ with $P \vdash_{\mathrm{SLDNF}} A$. Then $P \models A$. If $\gamma_{P}=\omega$, then $P \vdash_{\mathrm{SLDNF}} A$ if and only if $P \models A$. In particular, if $P$ is without local variables, then $P \models A$ if and only if $P \vdash_{\text {SLDNF }} A$.

Proof: By [5, Proposition 14.2], $M_{P}$ is the unique model of $\operatorname{comp}(P)$. By [5, Theorem 15.4], the first statement immediately holds. Now let $\gamma_{P}=\omega$ and $P \models A$. Then, by [1, Corollay 4.11], all SLDNFderivations of $P \cup\{\leftarrow A\}$ are finite and, therefore, $P \vdash_{\text {SLDNF }} A$ which proves the second statement. If $P$ is without local variables, then $P$ is acyclic by Proposition 2.2, which completes the proof using the second statement.

We establish next one of our main results: that every partial recursive function can be computed by an sld-program with cuts. We take the point of view (following [5]) that a cut does not affect the declarative semantics of a program. When talking about SLDNF-resolution for strictly level-decreasing programs with cuts, we assume that the selection function always selects the leftmost literal and, as discussed in [5], that the cut "prunes" the search tree. To obtain a well-defined procedural semantics of a given program, we assume that the topmost clause whose head unifies with a current goal is always selected first, as implemented in standard Prolog systems. So, for what follows, SLDNF-resolution is performed in the way just described.

For convenience, we will denote ground terms by lowercase letters and variables by uppercase letters when refering to a predicate. Thus, $p\left(x_{1}, \ldots, x_{n}, Y\right)$ means that all $x_{i}$ are ground and $Y$ is a variable. We write $(P, A) \vdash_{\mathrm{SLDNF}} B$ if $P \cup\{\leftarrow A\}$ has an answer substitution $\theta$ (via SLDNF-resolution) such that $A \theta=B$.

2.14 Theorem Identify $\mathbb{N}$ with the set $\left\{s^{n}(0) ; n \in \mathbb{N}\right\}$ by taking $s$ to be the successor function. Let $f$ be an $n$-ary partial recursive function. Then there exists a strictly level-decreasing program $P_{f}$ with cuts and an $(n+1)$-ary predicate symbol $p_{f}$ such that the following hold:

1. A call to $P_{f}$ with goal $p_{f}\left(x_{1}, \ldots, x_{n}, Y\right)$ or $p_{f}\left(x_{1}, \ldots, x_{n}, y\right)$ terminates via SLDNF-resolution if $\left(x_{1}, \ldots, x_{n}\right) \in \operatorname{dom}(f)$ and backtracking over the goal fails immediately.

2. $\left(P_{f}, p_{f}\left(x_{1}, \ldots, x_{n}, Y\right)\right) \vdash_{\mathrm{SLDNF}} p_{f}\left(x_{1}, \ldots, x_{n}, y\right)$ if and only if $\left(x_{1}, \ldots, x_{n}\right) \in \operatorname{dom}(f)$ and $f\left(x_{1}, \ldots, x_{n}\right)=y$.

3. For every $p_{f}\left(x_{1}, \ldots, x_{n}, y\right) \in B_{P}$ the following are equivalent:

(a) $P \models p\left(x_{1}, \ldots, x_{n}, y\right)$

(b) $P \vdash_{\text {SLDNF }} p\left(x_{1}, \ldots, x_{n}, y\right)$

(c) $f\left(x_{1}, \ldots, x_{n}\right)=y$.

Proof: We follow [13] and [5] with modifications where necessary. The proof is by induction on the number $q$ of applications of composition, primitive recursion, and minimalization needed to define $f$.

Suppose first that $q=0$. Thus $f$ must be either the zero function, the successor function, or a projection function.

Zero function

Suppose that $f$ is the zero function defined by $f(x)=0$. Define $P_{f}$ to be the program $p_{f}(X, 0) \leftarrow$. Successor function

Suppose that $f$ is the successor function defined by $f(x)=s(x)$. Define $P_{f}$ to be the program $p_{f}(X, s(X)) \leftarrow$. 


\section{Projection function}

Suppose that $f$ is the projection function defined by $f\left(x_{1}, \ldots, x_{n}\right)=x_{j}$ for some $j \in\{1, \ldots, n\}$. Define $P_{j}$ to be the program $p_{f}\left(X_{1}, \ldots, X_{n}, X_{j}\right) \leftarrow$.

Clearly, for each of the basic functions, the program $P_{f}$, as defined, is an sld-program with the desired properties.

Next, suppose that the partial recursive function $f$ is defined by $q>0$ applications of composition, primitive recursion, and minimalization.

\section{Composition}

Suppose that $f$ is defined by $f\left(x_{1}, \ldots, x_{n}\right)=h\left(g_{1}\left(x_{1}, \ldots, x_{n}\right), \ldots, g_{m}\left(x_{1}, \ldots, x_{n}\right)\right)$ where $g_{1}, \ldots, g_{m}$ and $h$ are partial recursive functions. By the induction hypothesis, corresponding to each $g_{i}$ (or $h$ ), there is an sld-program $P_{g_{i}}\left(P_{h}\right)$ with cuts and a predicate symbol $p_{g_{i}}\left(p_{h}\right)$ satisfying the conclusions of the theorem. We can suppose that the programs $P_{g_{1}}, \ldots, P_{g_{m}}, P_{h}$ do not have any predicate symbols in common. Define $P_{f}$ to be the union of these programs together with the clause

$$
p_{f}\left(X_{1}, \ldots, X_{n}, Z\right) \leftarrow p_{g_{1}}\left(X_{1}, \ldots, X_{n}, Y_{1}\right), \ldots, p_{g_{m}}\left(X_{1}, \ldots, X_{n}, Y_{m}\right), h\left(Y_{1}, \ldots, Y_{m}, Z\right), !
$$

Obviously, $P_{f}$ is an sld-program with cuts. Statement 1 is immediate under the assertion of the induction hypothesis, as is the 'if'-part of statement 2. The 'only-if' part is shown as in [5]. For statement 3, the equivalence of $3 \mathrm{a}$ and $3 \mathrm{c}$ is immediate and the equivalence of $3 \mathrm{~b}$ and $3 \mathrm{c}$ is shown in a manner analogous to that employed in [13].

\section{Primitive recursion}

Suppose that $f$ is defined by

$$
\begin{aligned}
f\left(x_{1}, \ldots, x_{n}, 0\right) & =h\left(x_{1}, \ldots, x_{n}\right) \\
f\left(x_{1}, \ldots, x_{n}, y+1\right) & =g\left(x_{1}, \ldots, x_{n}, y, f\left(x_{1}, \ldots, x_{n}, y\right)\right)
\end{aligned}
$$

where $h$ and $g$ are partial recursive functions. By the induction hypothesis, corresponding to $h$ (resp. $g$ ), there is an sld-program $P_{h}$ (resp. $P_{g}$ ) with cuts and a predicate symbol $p_{h}$ (resp. $p_{g}$ ) satisfying the conclusions of the theorem. We can also suppose that $P_{h}$ and $P_{g}$ do not have any predicate symbols in common. Define $P_{f}$ to be the union of $P_{h}$ and $P_{g}$ together with the clauses

$$
\begin{aligned}
p_{f}\left(X_{1}, \ldots, X_{n}, 0, Z\right) & \leftarrow p_{h}\left(X_{1}, \ldots, X_{n}, Z\right), ! \\
p_{f}\left(X_{1}, \ldots, X_{n}, s(Y), Z\right) & \leftarrow p_{f}\left(X_{1}, \ldots, X_{n}, Y, U\right), p_{g}\left(X_{1}, \ldots, X_{n}, Y, U, Z\right), !
\end{aligned}
$$

Obviously, $P_{f}$ is an sld-program with cuts. The desired properties are proven along the same lines as for composition.

\section{Minimalization}

Suppose that $f$ is defined by $f\left(x_{1}, \ldots, x_{n}\right)=\mu y\left(g\left(x_{1}, \ldots, x_{n}, y\right)=0\right)$ where $g$ is a partial recursive function. By the induction hypothesis, corresponding to $g$ there is an sld-program $P_{g}$ with cuts and a predicate symbol $p_{g}$ satisfying the conclusions of the theorem. Define $P_{f}$ to be $P_{g}$ together with the clauses

$$
\begin{aligned}
p_{f}\left(X_{1}, \ldots, X_{n}, 0\right) & \leftarrow p_{g}\left(X_{1}, \ldots, X_{n}, 0,0\right), ! \\
p_{f}\left(X_{1}, \ldots, X_{n}, s(Z)\right) & \leftarrow r\left(X_{1}, \ldots, X_{n}, Z\right), p_{g}\left(X_{1}, \ldots, X_{n}, s(Z), 0\right), ! \\
r\left(X_{1}, \ldots, X_{n}, 0\right) & \leftarrow \neg p_{g}\left(X_{1}, \ldots, X_{n}, 0,0\right) . \\
r\left(X_{1}, \ldots, X_{n}, s(Z)\right) & \leftarrow r\left(X_{1}, \ldots, X_{n}, Z\right), \neg p_{g}\left(X_{1}, \ldots, X_{n}, s(Z), 0\right) .
\end{aligned}
$$


Obviously, $P_{f}$ is an sld-program with cuts. Again, statements 1 and 2 are proven along the same lines as for composition by taking into account the fact that, if $p_{g}$ occurs in a subgoal of the computation, it is always ground. Note that $r\left(x_{1}, \ldots, x_{n}, z\right) \in M_{P_{f}}$ if and only if $\left(x_{1}, \ldots, x_{n}, k\right) \in \operatorname{dom}(g)$ and $g\left(x_{1}, \ldots, x_{n}, k\right) \neq 0$ for every $k<z$, and that the goal $r\left(x_{1}, \ldots, x_{n}, Z\right)$ subsequently yields all answer substitutions $Z / z$ $(z=0,1,2, \ldots)$ with $\left(x_{1}, \ldots, x_{n}, k\right) \in \operatorname{dom}(g)$ and $g\left(x_{1}, \ldots, x_{n}, k\right) \neq 0$ for all $k<z$, which yields the equivalence of $3 \mathrm{~b}$ and $3 \mathrm{c}$. To show the equivalence of $3 \mathrm{a}$ and $3 \mathrm{c}$, note that $P \models r\left(x_{1}, \ldots, x_{n}, z\right)$ if and only if $P \not \models p_{g}\left(x_{1}, \ldots, x_{n}, k, 0\right)$ for all $k<z$. So $P \models p_{f}\left(x_{1}, \ldots, x_{n}, z\right)$ if and only if $P \models p_{g}\left(x_{1}, \ldots, x_{n}, z, 0\right)$ and $P \forall \forall p_{g}\left(x_{1}, \ldots, x_{n}, k, 0\right)$ for all $k<z$. Now suppose $f\left(x_{1}, \ldots, x_{n}\right)=z$. Then by the induction hypothesis, the above yields that $P \models p_{f}\left(x_{1}, \ldots, x_{n}, z\right)$. Now suppose $f\left(x_{1}, \ldots, x_{n}\right) \neq z$. We consider three cases:

(1) $g\left(x_{1}, \ldots, x_{n}, z\right) \neq 0$. Then $P \not \models p_{f}\left(x_{1}, \ldots, x_{n}, z\right)$ immediately.

(2) $g\left(x_{1}, \ldots, x_{n}, k\right)=0$ for some $k<z$. Again $P \not \models p_{f}\left(x_{1}, \ldots, x_{n}, z\right)$ immediately.

(3) $\left(x_{1}, \ldots, x_{n}, k\right) \notin \operatorname{dom}(g)$ for some $k<z$. Then $r\left(x_{1}, \ldots, x_{n}, k\right)$ occurs as a subgoal of the computation and, therefore, so does $p_{g}\left(x_{1}, \ldots, x_{n}, k, 0\right)$. Note that $g$ cannot be one of the basic functions since they are total. For the same reason, $g$ cannot be defined by using composition and primitive recursion on the basic functions only. Consequently, at some point in the computation, a subgoal $p_{f_{0}}\left(x_{1}, \ldots, x_{n}, y\right)$ or $p_{f_{0}}\left(x_{1}, \ldots, x_{n}, Y\right)$ occurs with $f_{0}\left(x_{1}, \ldots, x_{n}\right)=\mu v\left(g_{0}\left(x_{1}, \ldots, x_{n}, y\right)=0\right)$ and $\left(x_{1}, \ldots, x_{n}\right) \notin \operatorname{dom}\left(f_{0}\right)$. There are two subcases to consider:

(i) $g\left(x_{1}, \ldots, x_{n}, m\right) \neq 0$ for all $m \in \mathbb{N}$. It is easily seen that in this case $P_{f_{0}}$ will not terminate on the subgoal $p_{f_{0}}\left(x_{1}, \ldots, x_{n}, Y\right)$ and will fail on the subgoal $p_{f_{0}}\left(x_{1}, \ldots, x_{n}, y\right)$.

(ii) $\left(x_{1}, \ldots, x_{n}, m\right) \notin \operatorname{dom}\left(g_{0}\right)$ for some $m \in \mathbb{N}$. The condition of this case is exactly as in case (3).

Thus, the argument can be repeated. Since every partial recursive function is defined by using minimalization only finitely often, the conclusion follows by induction.

Theorem 2.14 shows that sld-programs with cuts are computationally adequate with respect to SLDNFresolution as interpreter. It is ongoing work of the authors to investigate the possibilities of developing an alternative interpreter for sld-programs without cuts, based on SLDNF-resolution, which applies inductive methods where necessary.

\section{Logic Programs and Dynamical Systems}

In this part of the paper, we are going to explore in more detail certain remarks we made in [11] which relate logic programming semantics and dynamical systems. In fact we start by considering, in the context of logic programming, the use of the Hausdorff metric associated with a given metric and connections between logic programming semantics and the Vietoris space of $B_{P}$.

\subsection{Vietoris Space of $B_{P}$}

Let $(M, \rho)$ be a metric space. If $E, F$ are subsets of $M$, see [16], we define the distance between $E$ and $F$ to be $\rho(E, F)=\inf \{\rho(x, y) ; x \in E, y \in F\}=\rho(F, E)$; in case $E$ has only one point $x$, say, we write $\rho(x, F)$ for $\rho(\{x\}, F)$, with a similar convention if $F$ has only one point. Next, let $\mathcal{F}(M)$ denote the set of all non-empty closed subsets of $M$. For $A, B \in \mathcal{F}(M)$, define $d_{A}(B)=\max \{\rho(A, y) ; y \in B\}=$ $\max \{\min \{\rho(x, y) ; x \in A\} ; y \in B\}$. Finally, we define the Hausdorff metric $d$ on $\mathcal{F}(M)$ determined by $\rho$ by setting $d(A, B)=\max \left\{d_{A}(B), d_{B}(A)\right\}$. 
For the duration of this subsection, we will suppose that $\mathcal{L}$ contains at least one function symbol, so that $B_{P}$ is denumerable. This condition is not essential, but without it the following discussion becomes rather trivial from the topological viewpoint. With this assumption, we may choose a listing $B_{P}=\left\{A_{0}, A_{1}, A_{2}, \ldots\right\}$, of $B_{P}$. Now embed $B_{P}$ into the unit interval $[0,1]$ by defining $i\left(A_{0}\right)=0$ and $i\left(A_{n}\right)=2^{-n}$ for $n \geq 1$. Thus, $B_{P}$ becomes a compact metric space relative to the metric $\rho$ defined by setting $\rho\left(A_{n}, A_{m}\right)=\left|i\left(A_{n}\right)-i\left(A_{m}\right)\right|$. Let $V_{P}$ denote the subspace of $I_{P}$ consisting of all those elements of $I_{P}$ which contain $A_{0}$. Finally, we recall that the Vietoris space of a topological space $X$ is the set of all non-empty compact subsets of $X$ endowed with the Vietoris (or finite) topology, see [2,14], and is one of the standard hyperspaces studied in domain theory. In fact, when $X$ is a metric space, the Vietoris topology on $X$ coincides with the topology induced by the Hausdorff metric determined by the metric on $X$.

3.1 Theorem (1) A subset $I$ of $B_{P}$ is closed iff it is finite or contains $A_{0}$.

(2) The set $V_{P}$ is a closed, and therefore compact, subset of $I_{P}$ relative to the topology $Q$, and each element of $V_{P}$ is a non-empty closed subset of $B_{P}$.

(3) The topology of $V_{P}$, as a subspace of $I_{P}$ endowed with the topology $Q$, coincides with that induced by the Hausdorff metric determined by the metric $\rho$ on $B_{P}$. Therefore, $V_{P}$ is a closed subspace of the Vietoris space of $B_{P}$.

Proof: The details of (1) are elementary and are omitted. For (2), suppose that $I_{n} \in V_{P}$ for each $n \in \mathbb{N}$ and that $I_{n} \rightarrow I$ in $Q$. Since $A_{0} \in I_{n}$ for each $n, A_{0}$ is eventually in $I_{n}$. Therefore, $A_{0} \in I$ by the convergence criterion so that $I \in V_{P}$ as required. That each element of $V_{P}$ is a closed subset of $B_{P}$ follows from (1).

To establish (3), consider the identity map $j: V_{P}^{1} \rightarrow V_{P}^{2}$, in which $V_{P}^{1}$ denotes $V_{P}$ endowed with the subspace topology in $Q$, and $V_{P}^{2}$ denotes $V_{P}$ endowed with the Hausdorff metric $d$ determined by $\rho$. Since $V_{P}^{1}$ is compact and $V_{P}^{2}$ is Hausdorff, it suffices to show that $j$ is continuous. To do this, suppose that $I_{n} \in V_{P}$ for all $n \in \mathbb{N}$ and that $I_{n} \rightarrow I$ in $Q$, so that $I \in V_{P}^{1}$ by (2) of the theorem. We need to show that $d\left(I_{n}, I\right) \rightarrow 0$ as $n \rightarrow \infty$ and hence we need to show that both $d_{I_{n}}(I) \rightarrow 0$ and $d_{I}\left(I_{n}\right) \rightarrow 0$ as $n \rightarrow \infty$.

Consider $d_{I_{n}}(I)=\max \left\{\rho\left(I_{n}, y\right) ; y \in I\right\}=\max \left\{\min \left\{\rho(x, y) ; x \in I_{n}\right\} ; y \in I\right\}$. Let $\epsilon>0$ be given, and choose $n_{0} \in \mathbb{N}$ so large that $2^{-n_{0}} \leq \epsilon$. From the fact that $I_{n} \rightarrow I$ in $Q$, by the convergence criterion and by considering the finitely many elements $A_{1}, \ldots, A_{n_{0}}$, there is $k_{0} \in \mathbb{N}$ so large that, for $m=1, \ldots, n_{0}$, we have:

$$
\text { if } A_{m} \in I, \text { then for all } n \geq k_{0}, A_{m} \in I_{n}
$$

and

if $A_{m} \notin I$, then for all $n \geq k_{0}, A_{m} \notin I_{n}$

By Equation (2), we now have that, for $n \geq k_{0}, \min \left\{\rho(x, y) ; x \in I_{n}\right\}=0$ for any $y \in I \cap$ $\left\{A_{1}, \ldots, A_{n_{0}}\right\}$. Hence, for $n \geq k_{0}$, we have $\max \left\{\min \left\{\rho(x, y) ; x \in I_{n}\right\} ; y \in I\right\}=\max \{\min \{\rho(x, y) ; x \in$ $\left.I_{n}\right\} ; y \in I$ and $y=A_{n_{0}+m}$ for some $\left.m \geq 1\right\}$. But $\min \left\{\rho(x, y) ; x \in I_{n}\right\} \leq \rho\left(A_{0}, y\right)=i(y)$, since $A_{0} \in I_{n}$ for every $n$. Hence, for $n \geq k_{0}$, we have $\max \left\{\min \left\{\rho(x, y) ; x \in I_{n}\right\} ; y \in I\right.$ and $y=A_{n_{0}+m}$ for some $m \geq$ $1\} \leq \max \left\{i(y) ; y \in I\right.$ and $y=A_{n_{0}+m}$ for some $\left.m \geq 1\right\} \leq 2^{-\left(n_{0}+1\right)}<\epsilon$. Therefore, as $n \rightarrow \infty$, $d_{I_{n}}(I) \rightarrow 0$, as required.

Now consider $d_{I}\left(I_{n}\right)=\max \left\{\rho(x, I) ; x \in I_{n}\right\}=\max \left\{\min \{\rho(x, y) ; y \in I\} ; x \in I_{n}\right\}$. Again, with $\epsilon>0$ given, and taking the meaning already established for $n_{0}$ and $k_{0}$, let $n \geq k_{0}$. Suppose that $x=A_{m}$ , that $x \in I_{n}$ and that $m$ is any one of the values $1, \ldots, n_{0}$. Then $y=x$ must also belong to $I$. Otherwise, if $A_{m} \notin I$, then by Equation (3) we see that, for all $n \geq k_{0}$, we have $x \notin I_{n}$, which is contrary to 
the choice of $x \in I_{n}$ with $n \geq k_{0}$. Hence, again we see that $\min \{\rho(x, y) ; y \in I\}=0$ for each $x=$ $A_{m} \in I_{n}$ with $m=1, \ldots, n_{0}$ and $n \geq k_{0}$. Thus, for $n \geq k_{0}$, we have $\max \{\min \{\rho(x, y) ; y \in I\} ; x \in$ $\left.I_{n}\right\}=\max \left\{\min \{\rho(x, y) ; y \in I\} ; x \in I_{n}\right.$ and $x=A_{n_{0}+m}$ for some $\left.m \geq 1\right\}$. But $\min \{\rho(x, y) ; y \in I\} \leq$ $\rho\left(x, A_{0}\right)=i(x)$, since $A_{0} \in I$. Thus, for $n \geq k_{0}$, we have $\max \left\{\min \{\rho(x, y) ; y \in I\} ; x \in I_{n}\right.$ and $x=$ $A_{n_{0}+m}$ for some $\left.m \geq 1\right\} \leq \max \left\{i(x) ; x \in I_{n}\right.$ and $x=A_{n_{0}+m}$ for some $\left.m \geq 1\right\} \leq 2^{-\left(n_{0}+1\right)}<\epsilon$. Therefore, as $n \rightarrow \infty$, we have $d_{I}\left(I_{n}\right) \rightarrow 0$, as required.

3.2 Remark In relation to (2) of Theorem 3.1 it is quite possible in general for a sequence $I_{n}$ of closed sets to converge in $Q$ to a non-closed set. For example, the sequence in which $I_{n}=\left\{A_{1}, \ldots, A_{n}\right\}$, for each $n$, is a sequence of closed sets converging in $Q$ to $\left\{A_{1}, A_{2}, A_{3}, \ldots\right\}$, which is not closed.

Suppose now that $P$ satisfies the condition that it contains at least one unit clause, but is otherwise arbitrary and, in particular, it is not required that $T_{P}$ be continuous in $Q$ for what follows. This condition just imposed is quite mild and it implies in particular that $T_{P}(\emptyset) \neq \emptyset$. Moreover, it means that we can arrange matters so that $A_{0} \in T_{P}(I)$ for every $I \in I_{P}$. Therefore, $V_{P}$ is an invariant set under $T_{P}$ in the sense that $T_{P}\left(V_{P}\right) \subseteq V_{P}$, and we obtain the following corollary to Theorem 3.1.

3.3 Corollary Suppose that $P$ is any normal logic program which contains at least one unit clause, and that $A_{0}$ is chosen so that $A_{0} \in T_{P}(I)$ for every $I \in I_{P}$. Then, $T_{P}: V_{P} \rightarrow V_{P}$. Moreover, since $I_{1}=T_{P}(I)$ belongs to $V_{P}$ for any $I \in I_{P}$, iterates of $I$ enter and stay within $V_{P}$. Therefore, any fixed point of $T_{P}$ can be found within $V_{P}$.

In the sense that $T_{P}$ is not usually induced by a point map on $B_{P}$, we see that $T_{P}: V_{P} \rightarrow V_{P}$ is an abstract dynamical system.

3.4 Example The question clearly arises of providing syntactic conditions under which $T_{P}$ is a contraction mapping relative to the Hausdorff metric. For example, the "natural numbers" program $P$ as follows:

$$
\begin{aligned}
p(o) & \leftarrow \\
p(s(x)) & \leftarrow p(x)
\end{aligned}
$$

has the property that $T_{P}$ is such a contraction with the obvious listing of $B_{P}$. Indeed, $T_{P}$ has contractivity factor of $2^{-1}$, and we sketch the details of this next.

Let $I_{1}, I_{2} \in V_{P}$ with $I_{1} \neq I_{2}$. Since $I_{1}$ and $I_{2}$ contain $p(o)$, both $T_{P}\left(I_{1}\right)$ and $T_{P}\left(I_{2}\right)$ contain $p(o)$ and $p(s(o))$. Consider $d_{T_{P}\left(I_{1}\right)}\left(T_{P}\left(I_{2}\right)\right)=\max \left\{\min \left\{\rho(x, y) ; x \in T_{P}\left(I_{1}\right)\right\} ; y \in T_{P}\left(I_{2}\right)\right\}$. If $y$ is $p(o)$ or $p(s(o))$, then $x=y \in T_{P}\left(I_{1}\right)$ so that $\rho(x, y)=0$ in these cases and thus $\min \left\{\rho(x, y) ; x \in T_{P}\left(I_{1}\right)\right\}=0$. So suppose $y=p\left(s^{k}(o)\right) \in T_{P}\left(I_{2}\right)$ with $k \geq 2$. Then $\min \left\{\rho(x, y) ; x \in T_{P}\left(I_{1}\right)\right\}$ is achieved by some $x \in T_{P}\left(I_{1}\right)$ and is either $\left|2^{-l}-2^{-k}\right|$ for some $l \geq 1$ or is $\left|0-2^{-k}\right|$. Suppose the former case holds. Then there is $x=p\left(s^{l}(o)\right) \in T_{P}\left(I_{1}\right)$ with $l \geq k$. But then $p\left(s^{l-1}(o)\right) \in I_{1}, p\left(s^{k-1}(o)\right) \in I_{2}$ and $\min \left\{\rho(x, y) ; x \in T_{P}\left(I_{1}\right)\right\}=\left|2^{-l}-2^{-k}\right|=2^{-1}\left|2^{-(l-1)}-2^{-(k-1)}\right|=2^{-1} \min \left\{\rho\left(x, y^{\prime}\right) ; x \in I_{1}\right\}$ with $y^{\prime}=p\left(s^{k-1}(o)\right) \in I_{2}$. The case when $\min \left\{\rho(x, y) ; x \in T_{P}\left(I_{1}\right)\right\}=\left|0-2^{-k}\right|$ splits into two subcases each of which gives equalities of the form just derived, and therefore we see that $d_{T_{P}\left(I_{1}\right)}\left(T_{P}\left(I_{2}\right)\right)=2^{-1} d_{I_{1}}\left(I_{2}\right)$. The same sort of argument shows that $d_{T_{P}\left(I_{2}\right)}\left(T_{P}\left(I_{1}\right)\right)=2^{-1} d_{I_{2}}\left(I_{1}\right)$ and hence that $d\left(T_{P}\left(I_{1}\right), T_{P}\left(I_{2}\right)\right)=$ $2^{-1} d\left(I_{1}, I_{2}\right)$ as claimed. 
On the other hand, the "even numbers" program:

$$
\begin{aligned}
p(o) & \leftarrow \\
p(s(x)) & \leftarrow \neg p(x)
\end{aligned}
$$

does not have this property. For example, taking $I_{1}=\left\{p(o), p\left(s^{2}(o)\right), p\left(s^{5}(o)\right)\right\}$ and $I_{2}=B_{P}$, we find by direct calculation that $d\left(I_{1}, I_{2}\right)=2^{-2}=d\left(T_{P}\left(I_{1}\right), T_{P}\left(I_{2}\right)\right)$. Thus, $T_{P}$ is not in this case a contraction relative to the Hausdorff metric.

3.5 Remark Since $I_{P}$ is homeomorphic to the Cantor set, $T_{P}\left(V_{P}\right)$ can be thought of as a subset of the Cantor set and indeed is a compact subset if $T_{P}$ is continuous in $Q$. In view of the results of [15], which uses results of Moore to characterize Turing machines as subsets of the Cantor set, one may consider the extent to which $T_{P}\left(V_{P}\right)$ determines $P$ and the rôle, if any, of continuity of $T_{P}$.

\subsection{Iterated Function Systems}

We begin with a definition.

3.6 Definition An iterated function system (ifs), $\left\{X ; f_{1}, \ldots, f_{n}\right\}$, is given by a finite set of continuous functions $f_{i}: X \rightarrow X$, for $i=1, \ldots, n$, on a complete metric space $(X, d)$. The ifs is hyperbolic if each of the $f_{i}$ is a contraction mapping.

Now suppose that $P$ is an arbitrary normal logic program and that $P=P_{1} \cup \ldots \cup P_{n}$ is a partition of $P$ into $n$ sub-programs in which the definition of each predicate symbol is contained in one of the $P_{i}$ (the definition of a predicate symbol $p$ is the set of all clauses in $P$ in which the predicate symbol $p$ occurs in the head). We can then write $T_{P}$ as the union $\left(\bigcup_{i=1}^{n} T_{P_{i}}\right)$ in the sense that for all $I \in I_{P}$ we have $T_{P}(I)=\left(\bigcup_{i=1}^{n} T_{P_{i}}\right)(I)=\bigcup_{i=1}^{n} T_{P_{i}}(I)$. In this representation, each of the $T_{P_{i}}$ is to be thought of as a mapping of $I_{P}$ into itself rather than as a mapping of $I_{P_{i}}$ into itself.

3.7 Theorem Suppose that $P$ is partitioned as above. Then the following two statements hold.

(1) $T_{P}$ is continuous in $Q$ at a point $I \in I_{P}$ iff each of the $T_{P_{i}}$ is continuous in $Q$ at $I$.

(2) Suppose that each of the $T_{P_{i}}$ in the representation above is a contraction relative to Fitting's metric $d$ with contractivity factor $c_{i}=2^{-n_{i}}$, say, where $d$ is determined by the $\omega$-level mapping $l$. Then $T_{P}$ is a contraction relative to $d$ with contractivity factor $c=\max \left\{c_{i} ; i=1, \ldots, n\right\}$. Conversely, if $T_{P}$ is a contraction with factor of contractivity $c$ relative to $d$, then each of the $T_{P_{i}}$ is a contraction relative to $d$ with contractivity factor $\leq c$.

Note. In fact, the necessity of (1) and the first conclusion of (2) both hold relative to an arbitrary partition of $P$, as the proofs given below demonstrate.

Proof: (1) Suppose that each of the $T_{P_{i}}$ is continuous in $Q$ at $I$, and that $I_{m} \rightarrow I$ is an arbitrary sequence converging in $Q$ to $I$. Since $T_{P_{i}}\left(I_{m}\right) \rightarrow T_{P_{i}}(I)$ for each $i$, it is an easy consequence of the convergence criterion that $\bigcup_{i=1}^{n} T_{P_{i}}\left(I_{m}\right) \rightarrow \bigcup_{i=1}^{n} T_{P_{i}}(I)$ i.e. that $T_{P}\left(I_{m}\right) \rightarrow T_{P}(I)$, and so $T_{P}$ is continuous in $Q$ at $I$. Conversely, suppose that $T_{P}$ is continuous in $Q$ at $I$, that $I_{m} \rightarrow I$ in $Q$ and that $k \in\{1, \ldots, n\}$ is fixed but arbitrary; we show that $T_{P_{k}}\left(I_{m}\right) \rightarrow T_{P_{k}}(I)$ i.e. that $T_{P_{k}}$ is continuous in $Q$ at $I$.

Suppose that $A \in T_{P_{k}}(I)$. Then $A \in \bigcup_{i=1}^{n} T_{P_{i}}(I)$ and hence $A \in T_{P}(I)$. Since $T_{P}\left(I_{m}\right) \rightarrow T_{P}(I)$, we see by the convergence criterion that eventually $A$ belongs to $T_{P}\left(I_{m}\right)$ and hence eventually belongs to $\bigcup_{i=1}^{n} T_{P_{i}}\left(I_{m}\right)$. By the nature of the partition $A \notin T_{P_{i}}(J)$ for any $J$ and any $i \neq k$. Therefore, $A$ is 
eventually in $T_{P_{k}}\left(I_{m}\right)$. Now suppose that $A \notin T_{P_{k}}(I)$. This case divides into two subcases, the first of which is that $A \notin \bigcup_{i=1}^{n} T_{P_{i}}(I)=T_{P}(I)$. Then immediately we have that $A$ eventually not in $T_{P}\left(I_{m}\right)$ and so $A$ eventually not in $T_{P_{k}}\left(I_{m}\right)$. For the second subcase, suppose that $A \in T_{P}(I)$. Thus, $A \in T_{P_{j}}(I)$ with $j \neq k$. But then immediately $A \notin T_{P_{k}}(J)$ for any $J$ by the nature of the partition. So $A$ eventually not in $T_{P_{k}}\left(I_{m}\right)$. Therefore, $T_{P_{k}}$ is continuous in $Q$ at $I$ by the convergence criterion, as required.

(2) Put $c=\max \left\{c_{i} ; i=1, \ldots, n\right\}=2^{-m}$, say, where $m \geq 1$. Let $I_{1}, I_{2} \in I_{P}$ be arbitrary with $I_{1} \neq I_{2}$, and suppose $d\left(I_{1}, I_{2}\right)=2^{-k}$. By hypothesis, we have $d\left(T_{P_{i}}\left(I_{1}\right), T_{P_{i}}\left(I_{2}\right)\right) \leq c_{i} d\left(I_{1}, I_{2}\right)$, for $i=1, \ldots, n$, and so certainly we have, for each $i$, that $d\left(T_{P_{i}}\left(I_{1}\right), T_{P_{i}}\left(I_{2}\right)\right) \leq c d\left(I_{1}, I_{2}\right)=2^{-m} \cdot 2^{-k}=2^{-(m+k)}$. Thus, for $i=1, \ldots, n, T_{P_{i}}\left(I_{1}\right)$ and $T_{P_{i}}\left(I_{2}\right)$ agree on all ground atoms of level $<m+k$. Consider $T_{P}\left(I_{1}\right)=$ $\bigcup_{i=1}^{n} T_{P_{i}}\left(I_{1}\right)$ and $T_{P}\left(I_{2}\right)=\bigcup_{i=1}^{n} T_{P_{i}}\left(I_{2}\right)$. Suppose that $A$ is an arbitrary ground atom with $l(A)<m+k$. If $A \in T_{P}\left(I_{1}\right)$, then $A \in T_{P_{j}}\left(I_{1}\right)$, say. Hence, $A \in T_{P_{j}}\left(I_{2}\right)$ and therefore $A \in T_{P}\left(I_{2}\right)$. Conversely, if $A \in T_{P}\left(I_{2}\right)$, then $A \in T_{P}\left(I_{1}\right)$ and so $T_{P}\left(I_{1}\right)$ and $T_{P}\left(I_{2}\right)$ agree on all ground atoms $A$ with $l(A)<m+k$. Therefore

$$
d\left(T_{P}\left(I_{1}\right), T_{P}\left(I_{2}\right)\right) \leq 2^{-(m+k)}=c d\left(I_{1}, I_{2}\right)
$$

and so $T_{P}$ is a contraction with contractivity factor $\leq \max \left\{c_{i} ; i=1, \ldots, n\right\}$. Since $c=c_{j}$ for some $j$, there is an atom $A$ with $l(A)=m+k$ and a pair $I_{1}, I_{2} \in I_{P}$ such that $T_{P_{j}}\left(I_{1}\right)$ and $T_{P_{j}}\left(I_{2}\right)$ differ at $A$. But then $T_{P}\left(I_{1}\right)$ and $T_{P}\left(I_{2}\right)$ differ at $A$, by the conditions on the partition of $P$, so that $c$ cannot be reduced in Equation (4). In other words, the contractivity factor equals $c$, as required.

Conversely, suppose that $T_{P}$ is a contraction with contractivity factor $c=2^{-m}$. Suppose that $I_{1}, I_{2} \in I_{P}$ are arbitrary and that $d\left(I_{1}, I_{2}\right)=2^{-k}$. Then $d\left(T_{P}\left(I_{1}\right), T_{P}\left(I_{2}\right)\right) \leq c d\left(I_{1}, I_{2}\right)=2^{-(m+k)}$. Thus, $T_{P}\left(I_{1}\right)$ and $T_{P}\left(I_{2}\right)$ agree on all ground atoms of level $<m+k$. Fix $i=j \in\{1, \ldots, n\}$. Suppose that $A$ is an arbitrary ground atom with $l(A)<m+k$. If $A \in T_{P_{j}}\left(I_{1}\right)$, then $A \in T_{P}\left(I_{1}\right)$ and hence $A \in T_{P}\left(I_{2}\right)$. Again, by the conditions on the partition of $P$, this means that $A \in T_{P_{j}}\left(I_{2}\right)$. Since the converse also holds, we now see that $T_{P_{j}}\left(I_{1}\right)$ and $T_{P_{j}}\left(I_{2}\right)$ agree on all ground atoms of level $<m+k$. Therefore, $d\left(T_{P_{j}}\left(I_{1}\right), T_{P_{j}}\left(I_{2}\right)\right) \leq 2^{-(m+k)}=c d\left(I_{1}, I_{2}\right)$. It follows that $T_{P_{j}}$ is a contraction with contractivity factor $\leq c$, as required.

Thus, whenever $T_{P}$ is continuous in $Q,\left\{I_{P} ; T_{P_{1}}, \ldots, T_{P_{n}}\right\}$ is an iterated function system which is in fact hyperbolic under the conditions of Theorem 3.7 (2).

3.8 Example In the program $P$ :

$$
\begin{aligned}
q(o) & \leftarrow \\
q\left(s^{3}(x)\right) & \leftarrow p(x) \\
p(o) & \leftarrow \\
p\left(s^{2}(x)\right) & \leftarrow \neg p(x)
\end{aligned}
$$

the definition of $q$ has contractivity factor $\frac{1}{8}$, and the definition of $p$ has contractivity factor $\frac{1}{4}$. Therefore, $P$ determines a hyperbolic iterated function system with contractivity factor $\frac{1}{4}$.

Supposing, finally, that $T_{P}$ is continuous in $Q$, let $F\left(I_{P}\right)$ denote the set of non-empty compact subsets of $I_{P}$ endowed with the Hausdorff metric $d_{h}$ induced by $d$, where $d$ is the metric determined by a finite $\omega$ level mapping $l$. Then, in the standard way, $T_{P}$ induces a map $F_{P}: F\left(I_{P}\right) \rightarrow F\left(I_{P}\right)$ defined by $F_{P}(A)=$ $\left\{T_{P}(I) ; I \in A\right\}$ which is a contraction with contractivity factor $c$ if $T_{P}$ is such on $I_{P}$. Thus, $F\left(I_{P}\right)$ is the space of fractals over $I_{P}$ and $F_{P}$ is induced from the iterated function system $\left\{I_{P} ; T_{P_{1}}, \ldots, T_{P_{n}}\right\}$. 


\subsection{Generalized Iterated Function Systems}

In this final section, we show how the results of the previous section can be extended to arbitrary level mappings $l$ by using the generalized ultrametric $d_{l}$ in place of Fitting's ultrametric $d$. We let $B$ denote a countable set, let $D=2^{B}$ as defined in Section 2.1 and let $l: B \rightarrow \gamma$ be a level mapping where $\gamma>\omega$ is a successor ordinal.

3.9 Definition The orbit of $I \in D$ under a function $f: D \rightarrow D$ is defined to be the transfinite sequence $\left(f^{\alpha}(I)\right)_{\alpha<\gamma}$, where $f^{0}(I)=I, f^{\alpha}(I)=f\left(f^{\alpha-1}(I)\right)$ for every successor ordinal $\alpha<\gamma$ and $f^{\alpha}(I)=$ $\operatorname{gl}\left(\left(f^{\beta}(I)\right)_{\beta<\alpha}\right)$ for every limit ordinal $\alpha<\gamma$.

3.10 Proposition With the notation just established, the following statements hold.

(1) $\left(f^{\alpha}(I)\right)$ converges in $Q$ iff there exists some ordinal $\gamma_{0}$ such that $f^{\alpha}(I)=f^{\beta}(I)$ for all $\alpha, \beta \geq \gamma_{0}$.

(2) In the situation of (1), $f^{\gamma_{0}}(I)$ is a fixed point of $f$.

Proof: Suppose $\left(f^{\alpha}(I)\right)$ converges in $Q$. Then its limit is the set $J=\{a \in$ $B$; $a$ eventually belongs to $\left.\left.f^{\alpha}(I)\right)\right\}$. Let $a \in B$ be arbitrary. If $a \in J$, then there is an ordinal $\gamma_{a}<\gamma$ such that, for all $\alpha \geq \gamma_{a}$, we have $a \in f^{\alpha}(I)$. If $a \notin J$, then there is an ordinal $\delta_{a}<\gamma$ such that, for all $\alpha \geq \delta_{a}$, we have $a \notin f^{\alpha}(I)$. Set $\gamma_{0}=\sup \left\{\gamma_{a}, \delta_{a} ; a \in B\right\}<\gamma$. Then it is easy to see that $J=f^{\gamma_{0}}(I)=f^{\alpha}(I)$ for all $\alpha \geq \gamma_{0}$, from which the necessity of (1) follows and also (2).

Conversely, suppose $\gamma_{0}$ is an ordinal with the property stated in (1), and let $J$ denote the common value of the $f^{\beta}(I)$ for all $\beta \geq \gamma_{0}$. Then it is immediate that $\left(f^{\alpha}(I)\right) \rightarrow J$ in $Q$.

3.11 Definition In the situation of Proposition $3.10(1)$, we call $(D, l, f)$ a fixed-point triple relative to $I$.

3.12 Lemma Let $f: D \rightarrow D$ be strictly contracting with respect to $d_{l}$. Then the following statements hold. (1) For every ordinal $\alpha$, we have $d_{l}\left(f^{\alpha}(I), f^{\alpha+1}(I)\right) \leq 2^{-\alpha}$.

(2) Let $a \in B$ with $l(a)=\alpha$. Then either (i) for all $\beta>\alpha$, we have $a \in f^{\beta}(I)$, or (ii) for all $\beta>\alpha$, we have $a \notin f^{\beta}(I)$.

Proof: (1) We establish the assertion via transfinite induction. We may suppose that $f^{\alpha}(I)$ is not a fixed point of $f$, for that case is trivial. Also, for $\alpha=0$ the proposition trivially holds. So suppose the proposition holds for all ordinals less than $\alpha$. If $\alpha=\beta+1$ is a successor ordinal, we have $d_{l}\left(f^{\alpha}(I), f^{\alpha+1}(I)\right)<d_{l}\left(f^{\beta}(I), f^{\beta+1}(I)\right) \leq 2^{-\beta}$. Therefore, $d_{l}\left(f^{\alpha}(I), f^{\alpha+1}(I)\right)<2^{-(\alpha-1)}$ and hence $d_{l}\left(f^{\alpha}(I), f^{\alpha+1}(I)\right) \leq 2^{-\alpha}$ as required. If $\alpha$ is a limit ordinal, we have to show that $d_{l}\left(\operatorname{gl}\left(\left(f^{\beta}(I)\right)_{\beta<\alpha}\right), f\left(\operatorname{gl}\left(\left(f^{\beta}(I)\right)_{\beta<\alpha}\right)\right) \leq 2^{-\alpha}\right.$. By construction of the greatest limit and the induction hypothesis, we have $d_{l}\left(\operatorname{gl}\left(\left(f^{\beta}(I)\right)_{\beta<\alpha}\right), f^{\beta_{0}}(I)\right) \leq 2^{-\beta_{0}}$ for all $\beta_{0}<\alpha$. Hence, for every successor ordinal $\beta_{0}=\beta_{1}+1<\alpha$, we have $d_{l}\left(f\left(\operatorname{gl}\left(\left(f^{\beta}(I)\right)_{\beta<\alpha}\right)\right), f^{\beta_{0}}(I)\right)<d_{l}\left(\operatorname{gl}\left(\left(f^{\beta}(I)\right)_{\beta<\alpha}\right), f^{\beta_{1}}(I)\right) \leq 2^{-\beta_{1}}<2^{-\beta_{0}}$. Since $d_{l}$ is an ultrametric, we get $d_{l}\left(\operatorname{gl}\left(\left(f^{\beta}(I)\right)_{\beta<\alpha}\right), f\left(\operatorname{gl}\left(\left(f^{\beta}(I)\right)_{\beta<\alpha}\right)\right)\right) \leq 2^{-\beta_{0}}$ for all $\beta_{0}<\alpha$, which suffices.

(2) The proof is by transfinite induction on $\beta$. We consider the case $a \in f^{\alpha+1}(I)$, and the case $a \notin f^{\alpha+1}(I)$ is analogous. We have to show that $a \in f^{\beta}(I)$ for all $\beta>\alpha$. Suppose this is true for all $\beta$ with $\alpha<\beta<\beta_{0}$. If $\beta_{0}=\beta_{1}+1$ is a successor ordinal, then $d_{l}\left(f^{\beta_{0}}(I), f^{\beta_{1}}(I)\right) \leq 2^{-\beta_{1}}$ and therefore $a \in f^{\beta_{0}}(I)$ in this case. If $\beta_{0}$ is a limit ordinal, then $a \in f^{\beta_{0}}(I)$ by definition of greatest limit. 
3.13 Theorem Let the function $f: D \rightarrow D$ be strictly contracting with respect to $d_{l}$. Then $(D, l, f)$ is a fixed-point triple relative to every $I \in D$.

Proof: By the previous lemma, $f^{\alpha}(I)$ converges in $Q$ for every $I \in D$.

The following corollary is immediate.

3.14 Corollary For every strictly level-decreasing program $P,\left(I_{P}, l, T_{P}\right)$ is a fixed-point triple relative to every $I \in I_{P}$.

For an injective level mapping, $D$ can be identified with the space of all transfinite sequences $\left(a_{\alpha}\right)_{\alpha<\gamma}$, where $a_{\alpha} \in \mathbf{2}$ for every $\alpha$ and $\mathbf{2}$ denotes the set $\{0,1\}$.

3.15 Proposition For every strictly level-decreasing program $P$ there exists an injective level mapping.

Proof: The construction from Defintion 2.1 easily adapts and we omit the details.

The following result gives a straightforward generalization of Theorem 3.7 (2).

3.16 Theorem Let $P$ be an arbitrary normal logic program, let $l: B_{P} \rightarrow \gamma$ be a level mapping and let $P=\bigcup_{\kappa \in K} P_{\kappa}$ be a partition of $P$ into subprograms. Then the following statements hold.

(1) If every $T_{P_{\kappa}}$ is strictly contracting with respect to $d_{l}$, then $T_{P}$ is strictly contracting with respect to $d_{l}$.

(2) If the partition of $P$ has the property that the definition of each predicate symbol is contained in one of the $P_{\kappa}$, then the converse of (1) holds.

(3) In the situation of (1) or (2), $\left(I_{P}, l, T_{P}\right)$ is a fixed-point triple relative to every $I \in I_{P}$.

Proof: (1) Let $I_{1}, I_{2} \in I_{P}$ with $d_{l}\left(I_{1}, I_{2}\right)=2^{-\alpha}$, say. We have to show that $T_{P}\left(I_{1}\right)$ and $T_{P}\left(I_{2}\right)$ agree on all ground atoms with level $\leq \alpha$. Let $A \in B_{P}$ with $l(A) \leq \alpha$. If $A \in T_{P}\left(I_{1}\right)$, then $A \in T_{P_{\kappa}}\left(I_{1}\right)$, say. By the hypothesis on $T_{P_{\kappa}}$ we have $A \in T_{P_{\kappa}}\left(I_{1}\right)$ and therefore $A \in T_{P}\left(I_{1}\right)$. Conversely, if $A \in T_{P}\left(I_{2}\right)$, then $A \in T_{P}\left(I_{1}\right)$ and so $T_{P}\left(I_{1}\right)$ and $T_{P}\left(I_{2}\right)$ agree on all ground atoms $A$ with $l(A) \leq \alpha$.

(2) Let $I_{1}, I_{2} \in I_{P}$ with $d_{l}\left(I_{1}, I_{2}\right)=2^{-\alpha}$. Then $T_{P}\left(I_{1}\right)$ and $T_{P}\left(I_{2}\right)$ agree on all ground atoms of level $\leq \alpha$. Let $A \in B_{P}$ with $l(A) \leq \alpha$. If $A \in T_{P_{\kappa}}\left(I_{1}\right)$, then $A \in T_{P}\left(I_{1}\right)$ and hence $A \in T_{P}\left(I_{2}\right)$. By the hypothesis concerning the partition we get $A \in T_{P_{\kappa}}\left(I_{2}\right)$. Since the converse also holds, we now see that $T_{P_{\kappa}}\left(I_{1}\right)$ and $T_{P_{\kappa}}\left(I_{2}\right)$ agree on all ground atoms of level $\leq \alpha$, which suffices.

(3) This follows immediately from Theorem 3.13.

\section{Conclusions}

We have shown that the class of strictly level-decreasing logic programs is of special interest amongst all logic programs in that it has several rather pleasing properties, as follows. First, each program in this class has a unique supported model and therefore there is no argument about which model is "best" since all the standard models (perfect model, weakly perfect model, stable model etc.) coincide. Second, this class is computationally adequate in that there is a rather simple interpreter relative to which it can compute all the partial recursive functions. Finally, we have shown that there are interesting aspects of this class of programs which relate it to ideas of current interest in dynamical systems. Such ideas are actively being pursued in the context of domain theory and in real number computation by Edalat in [2] and in more recent work of his. The full extent of the connections between logic programming and these other areas just mentioned remains to be determined, and is actively under investigation by the present authors. 
Strictly Level-Decreasing Logic Programs

\section{References}

[1] Apt KR, Pedreschi D. Reasoning about termination of pure prolog programs. Information and Computation 1993; 106:109-157

[2] Edalat A. Dynamical systems, measures and fractals via domain theory. Information and Computation $1995 ; 120(1): 32-48$

[3] Fitting M. Metric methods: three examples and a theorem. J. Logic Programming 1994; 21 (3):113-127

[4] Hitzler P. Topology and logic programming semantics. Diplomarbeit in Mathematik, Universität Tübingen, 1998

[5] Lloyd JW. Foundations of logic programming, Second Edition. Springer-Verlag, Berlin, 1988

[6] Priess-Crampe S, Ribenboim P. Fixed points, combs and generalized power series. Abh. Math. Sem. Univ. Hamburg 1993; 63:227-244

[7] Priess-Crampe S, Ribenboim P. Ultrametric spaces and logic programming. Preprint, October 1997; pp 1-12

[8] Przymusinski T. On the declarative semantics of deductive databases and logic programs. In: Minker J (ed) Foundations of deductive databases and logic programming. Morgan Kaufmann Publishers Inc., Los Altos, 1988, pp 193-216

[9] Seda AK. Topology and the semantics of logic programs. Fundamenta Informaticae 1995; 24 (4):359386

[10] Seda AK. Quasi-metrics and the semantics of logic programs. Fundamenta Informaticae 1997; 29 (1):97-117

[11] Seda AK, Hitzler P. Topology and iterates in computational logic. In: Proceedings of the 12th Summer Conference on Topology and its Applications: Special Session on Topology in Computer Science, Ontario, August 1997; pp 1-43 (Topology Proceedings Vol 22. To appear)

[12] Seda AK, Hitzler P. Sur les programmes logiques lacalement stratifiés. C.R. Acad. Sc. Paris 1998; pp 1-5. To appear

[13] Šebelík J, Štěpánek P. Horn clause programs for recursive functions. In: Clark KL, Tärnlund S-Å (eds) Logic programming. Academic Press, New York, 1982, pp 324-340

[14] Smyth MB. Topology. In: Abramsky S, Gabbay DM, Maibaum TSE (eds) Handbook of logic in computer science, Vol. 1. Oxford Science Publications, Oxford University Press, 1992, pp 641-761

[15] Walsh J. Topology, fractals and domain theory - towards a geometria. M.Sc Thesis, University of Dublin, Trinity College, 1997

[16] Willard S. General topology. Addison Wesley Series in Mathematics, Addison Wesley Publishing Company Inc., Massachusetts, 1970 